\title{
Sympathetic Nerve Innervation and Metabolism in Ischemic Myocardium in Response To Remote Ischemic Conditioning
}

\section{Attila Kiss}

Center for biomedical research and translational surgery, Medical university of Vienna

\section{Xia Lu}

Department of Nuclear Medicine, Beijing Anzhen Hospital

\section{Michaela Schlederer}

Institute of Pathology, Medical University of Vienna

\section{Patrick M Pilz}

Center for Biomedical Research and Translation Surgery, Medical University of Vienna

\section{Petra Lujza Szabo}

Center for Biomedical Research and Translational Surgery

\section{Ping Wu}

Medical University of Vienna Department of Biomedical Imaging and Image-guided Therapy:

Medizinische Universitat Wien Universitatsklinik fur Radiologie und Nuklearmedizin

\section{Lukas Weber}

Center for Biomedical Research and Translation Surgery

\section{Chrysoula Vraka}

Medical University of Vienna Department of Biomedical Imaging and Image-guided Therapy:

Medizinische Universitat Wien Universitatsklinik fur Radiologie und Nuklearmedizin

\section{Verena Pichler}

University of Vienna: Universitat Wien

\section{Markus Mitterhauser}

Medical University of Vienna Department of Biomedical Imaging and Image-guided Therapy:

Medizinische Universitat Wien Universitatsklinik fur Radiologie und Nuklearmedizin

\section{Xiaoli Zhang}

Beijing An Zhen Hospital: Capital Medical University Affiliated Anzhen Hospital

\section{Dietmar Abraham}

Medical University of Vienna Centre for Anatomy and Cell Biology: Medizinische Universitat Wien

Zentrum fur Anatomie und Zellbiologie

\section{Bruno K Podesser}

Center for Biomedical Research and Translational Surgery

\section{Marcus Hacker}


Medical University of Vienna Department of Biomedical Imaging and Image-guided Therapy:

Medizinische Universitat Wien Universitatsklinik fur Radiologie und Nuklearmedizin

\section{Xiang Li ( $\nabla$ lixiangmed@gmail.com )}

Medical University of Vienna Department of Biomedical Imaging and Image-guided Therapy:

Medizinische Universitat Wien Universitatsklinik fur Radiologie und Nuklearmedizin

https://orcid.org/0000-0002-0016-6233

\section{Research Article}

Keywords: Myocardial ischemia/reperfusion, Remote ischemic preconditioning, Cardiac sympathetic nerve, Cardiac metabolism

Posted Date: December 29th, 2021

DOI: https://doi.org/10.21203/rs.3.rs-1193054/v1

License: (a) (1) This work is licensed under a Creative Commons Attribution 4.0 International License. Read Full License 


\section{Abstract \\ Background}

Multiple potential interventions have been tested to protect the heart against myocardial ischemia/reperfusion (MIR) injury. Remote ischemic conditioning (RIC), an endogenous cardioprotective approach, could markedly improve cardiac function post-myocardial ischemia injury. In this study, we aimed to assess the effects of RIC on cardiac sympathetic nerve innervation and metabolism in the association with Chondroitin sulfate proteoglycans (GSPG).

\section{Methods}

Transient myocardial ischemia (30 $\mathrm{min}$ ) is induced by ligature of the left anterior descending coronary artery ligation (LAD) in male Sprague Dawley rats (250-350 g), in vivo cardiac $\left[{ }^{11} \mathrm{C}\right] \mathrm{mHED}$ and $2-\left[{ }^{18} \mathrm{~F}\right] \mathrm{FDG}$ PET scans were performed at 14 days after ischemia. Remote ischemic preconditioning (RIPerc) was induced by three cycles of five-minute-long unilateral hind limb ischemia and intermittent five minutes of reperfusion during $L A D$ occlusion period. The quantitative parameters were quantified in parametric polar maps. This standardized format facilitates the regional radioactive quantification of parameters in deficit regions to remote areas. The ex vivo radionuclide distribution was additionally identified using autoradiography. Myocardial neuron density and GSPG expression were assessed by immunohistochemistry.

\section{Results}

There was no significant difference in the metabolism-defected to the remote activity ratio $(44.6 \pm 4.8 \%$ vs. $45.4 \pm 4.4 \%)$ between control rats (MIR) and treated (MIR+RIPerc) rats $(P>0.05)$. Additionally, the mean nervous activity of denervated myocardium activity was significantly elevated in rats with RIPerc coupled with reduced denervated myocardium size compared to the rats MIR group $(35.9 \pm 7.1 \%$ vs. $28.9 \pm 2.3 \%$ of the left ventricular $(L V)$ remote area $(P<0.05)$. These findings were associated with preserved $L V$ systolic function and a significant reduction in GSPG expression in the myocardium.

\section{Conclusion}

RIPerc presented the effect on cardiac sympathetic nerve innervation following ischemia, but there is no significant effect on myocardial metabolism. A long-term outcome study is warranted.

\section{Introduction}


Myocardial infarction (MI) followed by adverse cardiac remodeling and heart failure (HF) is a leading cause of mortality and morbidity ${ }^{[1,2]}$. Despite the robust development of early revascularization and pharmacological therapy, adverse cardiac remodeling leads to poor clinical outcomes in patients with MI

${ }^{[3]}$. Therefore, novel therapeutic strategies that effectively mitigate or reverse adverse post-infarcted cardiac remodeling and dysfunction remain challenging. Besides cardiomyocytes death due to acute $\mathrm{Ml}$, transmural Ml damages sympathetic nerve (SN) fibers running through the infarct zone and results in regional sympathetic denervation in the heart ${ }^{[4,5]}$. It is also known that the $\mathrm{SN}$ in the myocardium is more prone to suffer from ischemic insult than cardiomyocytes ${ }^{[6,7]}$.

Nerve injury causes the 'activation' of peripheral glia, the Schwann cells (SCs), whose repair-specific functions affect the whole microenvironment ${ }^{[8]}{ }^{[9]}$. These findings suggest that the local environment can manipulate SCs associated with nerves in injured organs to provide tissue-specific support. During regeneration of the heart muscle with its unique innervation, neuregulin-1 (NRG1) and Nerve growth factor $(N F G)$ are highly upregulated in the injured/infarcted heart ${ }^{[10]}\left[{ }^{111]}\right.$. Their regenerative effect has been primarily associated with cardiomyocyte proliferation and maybe nerve re-innervation, but NRG1 signaling may also be crucial for the proliferation, survival, motility, and differentiation of SCs following a myocardial injury such as $\mathrm{MI}^{[12]}$. In line with that, our group has demonstrated that remote ischemic perconditioning (RIPerc) markedly improved cardiac function and post-Ml adverse remodeling and this was associated with an increase of cardiac NRG-1 expression in the rat model of myocardial ischemia/reperfusion injury (MIR) ${ }^{[13]}$. However, whether RIPerc alleviates or not cardiac SN innervation following MIR is largely unknown.

Regional quantification of cardiac SN function, in conjunction with an assessment of regional viability, is essential for investigating ischemia injury. Cardiac positron emission tomography (PET) utilizing 2-deoxy2-[ $\left.{ }^{18} \mathrm{~F}\right]$ fluoro-D-glucose $\left(2-\left[{ }^{18} \mathrm{~F}\right] \mathrm{FDG}\right)$ showed superior sensitivity for myocardial metabolism assessment. Of importance, PET with the ${ }^{11} \mathrm{C}$-labeled norepinephrine analog $\left[{ }^{11} \mathrm{C}\right]$ meta-Hydroxyephedrine $\left(\left[{ }^{11} \mathrm{C}\right] \mathrm{mHED}\right)$ enables non-invasive idenfication of local cardiac SN injury in patients with HF and ischemic cardiomyopathy [14] [15] [16]. Altogether, these reports indicate that the delay of cardiac SN innervation in infarcted and peri-infarcted tissue may be considered an essential component and surrogate marker of post-MI adverse LV remodeling and sudden cardiac death. Therefore, utilizing PET imaging and developing novel pharmacological or non-pharmacological approaches for boosting early innervation may significantly reduce cardiac mortality in patients with MI.

\section{Methods}

\section{Animals}

Male Sprague-Dawley rats (10-12 weeks old, 250-300 g, Department for Laboratory Animal Science and Genetics, Himberg, Austria) were used. The experimental protocol was approved by the Regional Ethics Committee for Laboratory Animal Experiments at the Medical University of Vienna and the Austrian 
Ministry of Science Research and Economy (BMWFW-66.009/xxx-WF/V/3b/2016), and conforms with the Guide for the Care and Use of Laboratory Animals, published by the US National Institutes of Health (NIH Publication No. 85-23, revised 1996).

\section{Myocardial ischemia and reperfusion in vivo}

Myocardial ischemia was induced by ligation of the left coronary artery (LCA) for 30 min followed by 14 days of reperfusion as described previously in rats ${ }^{[13]}$. Briefly, rats were anesthetized by intraperitoneal injection of a mixture of Xylazine ( $4 \mathrm{mg} / \mathrm{kg}$; Bayer, Germany) and Ketamine (100 mg/kg; Dr E. Gräub AG, Switzerland), intubated (14-gauge tube) and ventilated ( $9 \mathrm{ml} / \mathrm{kg}$ body weight, $75-85 \mathrm{stroke} / \mathrm{min})$. To induce myocardial ischemia, the heart was exposed via a left thoracotomy and a ligature was placed around the LAD 2-3 mm away from the origin. Rectal temperature was measured and maintained at $37.5-38.5^{\circ} \mathrm{C}$ by a heated operating table. Myocardial ischemia was associated with the pallor of the myocardial area at risk and ST-elevation on ECG signal. Reperfusion was initiated following the 30 min of LAD occlusion by removal of the snare. RIPerc was induced by three cycles of 5 minutes of IR on hindlimb performed during myocardial ischemia as described previously ${ }^{[17]}$. Analgesia was initiated by intraperitoneal injection of Piritramide $(0.1 \mathrm{ml} / \mathrm{kg}$ body weight) preoperatively and Piritramide in drinking water was applied as a postoperative analgesic regimen ( 2 ampules of Piritramide with $30 \mathrm{ml}$ of Glucose $5 \%$ in $250 \mathrm{ml}$ water).

\section{Small Animal PET/CT Imaging}

A dedicated small animal $\mu \mathrm{PET} / \mu \mathrm{CT}$ system (Inveon, Siemens Medical Solutions Inc., Erlangen, Germay) was employed. All animals were maintained under anesthesia throughout the imaging acquisition. The ECG gated 30 min dynamic $\left[{ }^{11} \mathrm{C}\right] \mathrm{mHED}$ PET $(40-50 \mathrm{Mbq})$ and 60 min dynamic insulin-stimulated 2$\left[{ }^{18} \mathrm{~F}\right] \mathrm{FDG}$ PET (30-40 Mbq) acquisition was conducted meanwhile at tracer injection. All data were acquired in three-dimensional (3D) list-mode and sorted into a 3D sonogram of 16 frames per cardiac cycle. The sinogram was reconstructed into a $128 \times 128 \times 95$ voxel image by 2D-filtered back-projection image reconstruction using a ramp filter with the Nyquist limit ( 0.5 cycles/voxel) as the cut-off frequency. The voxel size equaled $0.43 \times 0.43 \times 0.80 \mathrm{~mm}$. Data were normalized and corrected for randoms, dead time, and decay. A 10 min attenuation correction transmission scan was additionally performed before the $\mu$ PET scan.

\section{Imaging analysis}

Late uptake defined the LV with bottle-brush sampling. Late myocardial uptake was averaged from 4 frames of data for each imaging 15 to $30 \mathrm{~min}$ after $\left[{ }^{11} \mathrm{C}\right] \mathrm{mHED}$ injection and 45 to $60 \mathrm{~min}$ after 2$\left[{ }^{18} \mathrm{~F}\right] \mathrm{FDG}$ injection. Using the volumetric sampling of the cardiac PET/CT scans, we defined myocardial radioactivity LV sectors and semi-automatically constructed radioactivity into cardiac metabolism and nerve polar maps. Each polar map was normalized to a region of interest (ROI) in the remote myocardium. The metabolic deficit area in the myocardium was defined as $2-\left[{ }^{18} \mathrm{~F}\right] \mathrm{FDG}$ uptake $\leq 60 \%$ of maximum activity. LV myocardium with nerve denervation was considered by a threshold of $\leq 60 \%$ of the 
maximum activity in the polar map. $\left[{ }^{11} \mathrm{C}\right] m \mathrm{HED}$ retention in the denervated segment was determined. Myocardial ROls were drawn at the mid-ventricular level, the blood pool was measured at the left atrial cavity. All $\mu \mathrm{PET}$ images were analyzed using the MunichHeart/NM software package.

\section{Autoradiography}

Ex vivo accumulation of myocardial $\left[{ }^{11} \mathrm{C}\right] \mathrm{mHED}$ was assessed using $20 \mu \mathrm{m}$ sections of sample tissues with a digital autoradiography system (Cyclone ${ }^{\circledR}$ Plus Storage Phosphor System; PerkinElmer). Myocardial area of tracer uptake defect was determined by a threshold of $50 \%$ of maximum myocardial activity in midventricular slices; the radioactivity values of the placed ROls were drawn on short-axis slices and then were expressed as background-corrected photostimulated luminescence units per area. Measured values were normalized to account for differences in the amount of tracer injected. Then the uptake activity of the defect area was calculated as percentages in relation to the remote area on the short-axis slice.

\section{Assessment of LV hemodynamic in vivo}

Next to $\mu \mathrm{PET} / \mu \mathrm{CT}$ Imaging, LV hemodynamic function was invasively monitored as described previously [18]. In brief, LV hemodynamic parameters were invasively measured. Rats were anesthetized by intraperitoneal injection of a mixture of Xylazin (4 mg/kg; Bayer, Germany) and Ketamin (100 mg/kg; Dr E. Gräub AG, Switzerland), intubated and ventilated. The chest was opened and a microtip catheter (SPR409, 2F, Millar Instruments, Houston, USA) was gently inserted into the LV chamber. Hemodynamic parameters such as LV systolic pressure (LVSP), LV end-diastolic pressure (LVEDP), and heart rate (HR) were continuously recorded on Labchart (v7.3.2, Powerlab System (8/30, both AD Instruments, Spechbach, Germany) connected to a Bridge Amplifier (Bridge Amp, ADInstruments, GmbH, Spechbach, Germany) and a Transducer Control unit (Model TC-510, Millar Instruments Inc., Houston, Texas, USA).

\section{Histology and immunochemistry}

Formalin-fixed paraffin-embedded LV cardiac tissue sections were hematoxylin and eosin (HE) stained. Streptavidin-biotin immunostaining for CD68 (1:100; mouse monoclonal, ED1, ab31630, Abcam, Cambridge, MA, USA), Tyrosine Hydroxylase (1:100; rabbit monoclonal, EP1533Y, Abcam, Cambridge, MA, USA), Chondroitin Sulfate protogylcan (CSPG; 1:500; mouse monoclonal, clone Cat-301, MAB5284, Merck, Darmstadt, Germany) were used as described previously ${ }^{[18]}$. Briefly, primary antibodies were detected with appropriate biotinylated secondary antibody (Vector Laboratories, Burlingame, CA) and horseradish peroxidase (HRP) conjugated streptavidin (Dako, Glostrup, Denmark), developed with 3,3'-

diaminobenzidine (DAB) (Vector Laboratories), counterstained with hematoxylin, dehydrated and mounted in DPX (Merck, Darmstadt, Germany). Tyrosine Hydroxylase was developed with 3-Amino-9ethylcarbazole (AEC) under visual control, counterstained with hematoxylin, and mounted with Aquatex (Merck, Darmstadt, Germany). Digitalized images were generated with a Nikon Eclipse 80i (Tokyo, Japan) microscope.

\section{Statistics}


Parametric data are presented as mean \pm SD. Uptake variables of animals with MIR and RIPerc were compared with unpaired Student t-tests (continuous data). All statistical analyses were performed with commercial software (SPSS 24). A value of $P$ less than 0.05 was considered statistically significant.

\section{Results}

\section{Animal}

Experimental rats were allocated into the following groups; 1$)$ MIR ( $n=14)$ and 2) RIPerc with three cycles of 5 minutes of IR of a hindlimb performed during myocardial ischemia (MIR+RIPerc, $n=15)$. Shamoperated animals (Sham, without LAD occlusion; $n=6$ ) served as controls. Fourteen days after the reperfusion, $\mu \mathrm{PET}$ scans were performed (function, viability etc), hemodynamic function was performed 2 days after $\mu \mathrm{PET}$ analysis in vivo and heart were taken for radioassay and immunohistochemistry, respectively.

\section{$\left[{ }^{11} \mathrm{C}\right] \mathrm{m}$ HED Imaging}

The metabolism (2-[18 F]FDG uptake) presenting average myocardial metabolism-deficit activity ratio was $44.6 \pm 4.8 \%$ of the LV remote area in rats (MIR) vs $45.4 \pm 4.4 \%$ of the LV remote area in rats (MIR+RIPerc) $(\mathrm{P}>0.05)$. Additionally, the mean nervous activity ratio $\left(\left[{ }^{11} \mathrm{C}\right] m \mathrm{mED}\right.$ uptake) of denervated myocardium activity was significantly elevated in rats with RIC in comparison to the MIR group ( $35.9 \pm 7.1 \%$ vs. $28.9 \pm 2.3 \%$ of the LV remote area $(P<0.05)$. (Fig. 1)

Furthermore, the animal cardiac polar map determined that the metabolism-deficit (2-[ $\left.{ }^{18} \mathrm{~F}\right] \mathrm{FDG}$ uptake) area was $15.26 \pm 2.47 \%$ of the total LV in rats (MIR) vs. $14.67 \pm 4.74 \%$ of the total LV in rats (MIR+RIPerc) $(P>0.05)$. Additionally, the denervated myocardium area of the $L V \%$ was significantly increased in rats with $\mathrm{MI}$ in comparison to MIR+RIPerc $(27.78 \pm 6.62 \%$ vs. $19.52 \pm 5.3 \%$ of the LV in rats; $P<0.05)$ (Fig. 2$)$. Quantitative cardiac polar map of viability (2-[18 F]FDG), and sympathetic innervation $\left[{ }^{11} \mathrm{C}\right] \mathrm{mHED}$ PET images from 2 representative subjects (MIR vs MIR+RIPerc) are presented in Figure 1.

Representative autoradiography was shown in Figure 3, ex vivo $\left[{ }^{11} \mathrm{C}\right] \mathrm{mHED}$ uptake ratios to the remote region were $0.29 \pm 0.11$ (MIR) and $0.37 \pm 0.08$ (MIR+RIPerc) $(p<0.01)$. (Fig. 3)

\section{Immunochemistry}

There is a more prominent reduction in TH-positive cells in MIR rats in comparison to RIPerc animals (Fig. 4). The reduction of cardiac neurons after MIR was shown to link a transient overexpression of CSPG in the animal hearts ${ }^{[19]}$. Therefore, we assessed immunochemistry staining for CSPG in Sham, MIR and Ml+RIPerc cardiac tissue samples. In comparison to Sham animals, the expression of CSPG was markedly increased in cardiac tissue samples from MIR rats (Fig. 5) particularly; its expression was higher in the infarcted area and border zone. In contrast, in rats treated with RIPerc, we observed that the CSPG expression was similar to the Sham group (Fig. 5). In addition, immunohistochemistry was 
performed to evaluate the influx of CD68+ macrophages. No CD68+ cells were detected in the Sham group (Fig. 5). In contrast, high numbers of CD68+ macrophages were present in the infarcted myocardial tissue of the MIR group, whereas RIPerc was associated with a reduced influx of CD68+ macrophages (Fig. 5)

\section{Left ventricular hemodynamic function}

Left ventricular hemodynamic function was invasively monitored and analyzed the HR, LVSP, LVEDP, and $\pm \mathrm{LVdp} / \mathrm{dtmax}$. No differences between the groups were observed in HR (Table 1). However, decreased LVSP, increased LVEDP, and reduced peak velocities of pressure change during isovolumic contraction $(+\mathrm{dP} / \mathrm{dt})$ and isovolumic relaxation $(-\mathrm{dP} / \mathrm{dt})$ in rats with MIR were observed in comparison to the sham group $(P<0.01$, respectively). These parameters were markedly improved by RIPerc (Table 1$)$.

Table 1. Left ventricular hemodynamic parameters

\begin{tabular}{|c|c|c|c|}
\hline Parameter/Group & Sham-OP & MIR & MIR+RIPerc \\
\hline $\mathrm{n}$ & 4 & 5 & 6 \\
\hline LVSP (mm Hg) & $117 \pm 5$ & $83 \pm 5^{\star \star \star}$ & $95 \pm 8^{\#}$ \\
\hline LVEDP (mm Hg) & $2.46 \pm 0.50$ & $5.70 \pm 1.49^{\star \star \star}$ & $4.18 \pm 0.63$ \\
\hline$+\mathrm{dP} / \mathrm{dt}(\mathrm{mm} \mathrm{Hg} \mathrm{-1)}$ & $7074 \pm 564$ & $4255 \pm 380^{\star \star \star}$ & $5139 \pm 588^{\#}$ \\
\hline$-\mathrm{dP} / \mathrm{dt}(\mathrm{mm} \mathrm{Hg} \mathrm{-1)}$ & $-5879 \pm 478$ & $-3125 \pm 873^{* *}$ & $-3827 \pm 669$ \\
\hline HR (bpm) & $258 \pm 28$ & $257 \pm 40$ & $244 \pm 28$ \\
\hline \multicolumn{4}{|c|}{$\begin{array}{l}\text { LVSP: left ventricular systolic pressure; LVEDP: left-ventricular end-diastolic pressure; }(+/-) \mathrm{dP} / \mathrm{dt} \text { : peal } \\
\text { velocities of pressure change; HR: heart rate. Values are mean } \pm \mathrm{SD} ;{ }^{* *} p<0.01 \text { and }{ }^{* \star} \mathrm{p}<0.001 \mathrm{Sham} \\
\text { vs MIR, and }{ }^{\#} p<0.05 \mathrm{MIR} \text { vs MIR+RIPerc. }\end{array}$} \\
\hline
\end{tabular}

\section{Discussion}

Alterations of cardiac nerve innervation in cardiac pathophysiological diseases have been increasingly noticed. Nevertheless, a therapeutic approach to reduce cardiac sympathetic dysfunction is limitedly available.

Cardiac sympathetic and parasympathetic innervation plays multiple roles in cardiac physiology and pathophysiology. There is large body of evidence that selective parasympathetic, eg. vagus nerve stimulation during acute myocardial infarction protects the heart against myocardial IR-injury and chronic stimulation of the vagus nerve has also benefit on post $\mathrm{MI}$ remodelling and chronic heart failure, respectively [20] [21] [22]. However, large clinical trials failed to show similar benefits in patients with congestive HF. 
On the other hand, sympathetic hyperinnervation or denervation in the myocardium are connected to the progression of cardiac dysfunction [23] [24]. Sympathetic denervation typically occurs in the infarcted myocardium and is associated with sudden cardiac death [25] [26]. Impaired innervation was also demonstrated in non-infarcted myocardium in patients with ischaemic and dilated cardiomyopathy [23]. Factors affecting SN integrity in infarcted and remote myocardium are largely unknown. Nevertheless, a recent pioneering study by Siebert et al ${ }^{[27]}$ demonstrated that CSPG expression in the infarcted myocardium delays the sympathetic innervation following myocardial infarction. Moreover, microvascular perfusion abnormalities, even in the absence of epicardial coronary artery disease, may also relate to sympathetic dysfunction ${ }^{[23]}$. Therefore, novel non-invasive methods are urgently needed to visualize cardiac sympathetic (re)innervation in cardiovascular disease and find novel thearupetic approaches to improve cardiac sympathetic dysfunction.

Multiple shreds of evidence demonstrated that a portion of dysinnervated but viable myocardium is essential of cardiac sympathetic homogenous denervation with scar attenuates cardiac remodeling, improves cardiovascular function, as well as sympathetic denervation at myocardial scar may effectively reduce arrhythmia burden [28] [29] [30] [31]. In contrast, denervated but viable myocardium adjacent to infarcted myocardium may be particularly arrhythmogenic and prone to induce ventricular fibrillation and substantial risk for sudden cardiac death ${ }^{[22]}$. Thus, identifying nerve denervation in viable myocardium is crucial in patients with ischemic cardiomyopathy and may also provide surrogate markers for assessing the risk for sudden death. Cardiac molecular imaging allows for the specific identification of synaptic biomarkers in the cardiac autonomic nervous system.

The present study investigated the therapeutic effect of remote ischemic conditioning on cardiac sympathetic nerve re-innervation following myocardial infarction by quantitative in vivo imaging. Previous studies have investigated the cardioprotective signaling of RIC against acute MIR-injury and preventing cell death of cardiomyocytes ${ }^{[32]}{ }^{[33]}$. Moreover, Gho et al. demonstrated for the first time that the ganglion blocker hexamethonium abolishes cardioprotection established by RIC, suggesting the functional importance of neural (autonomic) pathways mediating this phenomenon ${ }^{[34]}$. However, evidence is lacking of whether RIC acts on SN activity and innervation in the subacute or chronic phase of MI. Therefore, we first assessed ischemia-mediated SN degradation and myocardial viability defection in a rat model of MIR. Interestingly, RIC presented cardiac protection against sympathetic denervation but has no protecting effect on viable myocardial stunning. These findings demonstrate that the SN might be more susceptible to ischemia injury than the myocardial cells. The remaining viable myocardium may preserve elevated SN fibers under remote ischemic conditioning and substantially preserve cardiac function. In line with that, we found that the RIC group showed significantly higher LVSP and a tendency to improve diastolic dysfunction (LVEDP) compared to the MIR group. Of importance, sympathetic denervation is strongly associated with microvascular dysfunction, which is the basis of the progression of diastolic dysfunction [23] [35]. Therefore, improving cardiac sympathetic dysfunction and re-innervation following MI may have potential clinical benefits in patients suffering from ischemic heart disease. 
However, further studies need to clarify the interaction of RIC, microvascular dysfunction and sympathetic dysfunction /denervation in humans.

During acute myocardial ischemia, damage to cardiac sympathetic neurons results in the release of norepinephrine into the myocardial interstitial space ${ }^{[36]}$. It has been recognized that the extent of denervation exceeds infarction following coronary artery occlusion. The neuronal damage could be transient or irreversible, depending on the severity and duration of ischemia, and reversible SN dysfunction has been reported but is slow in patients after myocardial ischemia $[6,23,25]$.

Chronic elevation of $\mathrm{SN}$ activity in the myocardium is a key component of the altered signaling pathways that accompany cardiac remodeling, increasing the risk for sudden cardiac death resulting from hypertension and acute $\mathrm{MI}{ }^{[24,25]}$. However, the dysregulation and a decrease in SN activity play a pivotal

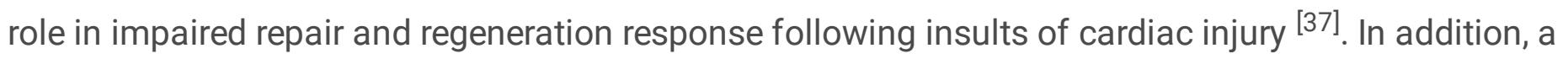
more recent study by Gardner et al ${ }^{[19]}$ suggests that early SN-reinnervation after MI reduced the risk for sudden cardiac death. Thereby therapeutic manipulation for boosting early SN innervation after MI may benefit LV remodeling, cardiac function and sudden cardiac death.

With injury or disease, an increase in CSPG expression is commonly observed close to lesioned areas [27]. However, these CSPG deposits form a substantial barrier to regeneration and are primarily responsible for the inability to repair damage in the brain and spinal cord. A more recent study showed that CSPG present in the cardiac scar after Ml prevents sympathetic innervation by binding the neuronal protein tyrosine phosphatase receptor $\sigma$ (PTP $\sigma$ ) and downstream signaling pathway activation may increase the risk for sudden cardiac death ${ }^{[19]}$. Interestingly, we also found that CSPG expression in the infarct and peri-infarct area was markedly increased in rats with MIR, but it expression was substantially decreased in RIPerc animals. This suggests that RIPerc may reduce the risk for sudden cardiac death via a mechanism involving the reduction in expression of CSPG and substantially boosting early sympathetic re-innervation post MI. In summary, sympathetic denervation in patients with ischemia and reperfusion has been extensively recognized, but the potential effect of reversible SN dysfunction is less widely appreciated.

\section{Limitations}

Certain limitations of our study need to be acknowledged. Due to gated cardiac perfusion, scans were not performed to determine the cardiac contraction due to high kinetic positron energy and consequently extensive positron range for small animal images. In addition, our study was not aimed to assess cardiac fibrosis and cardiac function by echocardiography or MRI following MIR. However, we have shown that RIPerc reduces cardiac fibrosis and improves hemodynamic function in comparison with untreated rats [13]

\section{Conclusion}


In this study, we found that remote ischemic perconditioning treatment boosted early innervation of cardiac SN following myocardial ischemia/reperfusion injury in association with the reduction in CSPG expression, whereas no net effects on the myocardial metabolism were observed.

\section{Declarations}

\section{Funding}

This work was supported by project No. $920 ̈$ u from Austria-Hungary Action Foundation (A.K) and by an unrestricted research grant of the Ludwig Boltzmann Society (REM2017-20, A.K. and B.K.P.). This article is based upon work from COST Action EU-CARDIOPROTECTION CA16225 supported by COST (European Cooperation in Science and Technology).

\section{Acknowledgments}

The authors thank Milat Inci (Ludwig Boltzmann Institute for Cardiovascular Research, Vienna, Austria) and Sandra Trojanek (Center for Anatomy and Cell Biology, Medical University Vienna, Austria) and Anna Zacher and Stefanie Ponti (Preclinical Imaging Laboratory, Medical University of Vienna) for technical assistance in laboratory works.

\section{Conflicts of Interest}

The authors declare no conflict of interest.

\section{References}

1. van der Laan AM, Nahrendorf M, Piek JJ. Healing and adverse remodelling after acute myocardial infarction: role of the cellular immune response. Heart. 2012;98(18):1384-90. doi:10.1136/heartjnl2012-301623.

2. White HD, Chew DP. Acute myocardial infarction. Lancet. 2008;372(9638):570-84. doi:10.1016/S0140-6736(08)61237-4.

3. Mozaffarian D, Benjamin EJ, Go AS, Arnett DK, Blaha MJ, Cushman M, Das SR, de Ferranti S, Després JP, Fullerton HJ, Howard VJ, Huffman MD, Isasi CR, Jiménez MC, Judd SE, Kissela BM, Lichtman JH, Lisabeth LD, Liu S, Mackey RH, Magid DJ, McGuire DK, Mohler ER 3rd, Moy CS, Muntner P, Mussolino ME, Nasir K, Neumar RW, Nichol G, Palaniappan L, Pandey DK, Reeves MJ, Rodriguez CJ, Rosamond W, Sorlie PD, Stein J, Towfighi A, Turan TN, Virani SS, Woo D, Yeh RW, Turner MB. Heart Disease and Stroke Statistics-2016 Update: A Report From the American Heart Association. Circulation. 2016;133(4):e38-60. doi:10.1161/cir.0000000000000350.

4. Barber MJ, Mueller TM, Henry DP, Felten SY, Zipes DP. Transmural myocardial infarction in the dog produces sympathectomy in noninfarcted myocardium. Circulation. 1983;67(4):787-96. doi:10.1161/01.cir.67.4.787. 
5. Dae MW, Herre JM, O'Connell JW, Botvinick EH, Newman D, Munoz L. Scintigraphic assessment of sympathetic innervation after transmural versus nontransmural myocardial infarction. J Am Coll Cardiol. 1991;17(6):1416-23. doi:10.1016/s0735-1097(10)80156-1.

6. Hartikainen J, Kuikka J, Mäntysaari M, Länsimies E, Pyörälä K. Sympathetic reinnervation after acute myocardial infarction. The American journal of cardiology. 1996;77(1):5-9. doi:10.1016/s00029149(97)89125-4.

7. Sisson JC, Johnson J, Bolgos G, Lynch JJ Jr, Uprichard A, Driscoll E, Wieland DM, Lucchesi B. Portrayal of adrenergic denervation in the presence of myocardial infarction: a feasibility study. American journal of physiologic imaging. 1990;5(4):151-66.

8. Clements MP, Byrne E, Camarillo Guerrero LF, Cattin AL, Zakka L, Ashraf A, Burden JJ, Khadayate S, Lloyd AC, Marguerat S, Parrinello S. The Wound Microenvironment Reprograms Schwann Cells to Invasive Mesenchymal-like Cells to Drive Peripheral Nerve Regeneration. Neuron. 2017;96(1):98114.e7. doi:10.1016/j.neuron.2017.09.008.

9. Barton MJ, John JS, Clarke M, Wright A, Ekberg J. The Glia Response after Peripheral Nerve Injury: A Comparison between Schwann Cells and Olfactory Ensheathing Cells and Their Uses for Neural Regenerative Therapies. Int J Mol Sci 2017;18(2). doi:10.3390/ijms18020287.

10. Gambarotta G, Fregnan F, Gnavi S, Perroteau I. Neuregulin 1 role in Schwann cell regulation and potential applications to promote peripheral nerve regeneration. International review of neurobiology. 2013;108:223-56. doi:10.1016/b978-0-12-410499-0.00009-5.

11. Aloe L, Rocco ML, Balzamino BO, Micera A. Nerve Growth Factor: A Focus on Neuroscience and Therapy. Current neuropharmacology. 2015;13(3):294-303. doi:10.2174/1570159x13666150403231920.

12. Britsch S. The neuregulin-I/ErbB signaling system in development and disease. Adv Anat Embryol Cell Biol. 2007;190:1-65.

13. Pilz PM, Hamza O, Gidlöf O, Gonçalves IF, Tretter EV, Trojanek S, Abraham D, Heber S, Haller PM, Podesser BK, Kiss A. Remote ischemic perconditioning attenuates adverse cardiac remodeling and preserves left ventricular function in a rat model of reperfused myocardial infarction. Int $\mathrm{J}$ Cardiol. 2019;285:72-9. doi:10.1016/j.ijcard.2019.03.003.

14. Mélon P, Schwaiger M. Imaging of metabolism and autonomic innervation of the heart by positron emission tomography. Eur J Nucl Med. 1992;19(6):453-64. doi:10.1007/bf00177375.

15. Boutagy NE, Sinusas AJ. Recent Advances and Clinical Applications of PET Cardiac Autonomic Nervous System Imaging. Curr Cardiol Rep. 2017;19(4):33. doi:10.1007/s11886-017-0843-0.

16. Aikawa T, Naya M, Obara M, Oyama-Manabe N, Manabe O, Magota K, Ito YM, Katoh C, Tamaki N. Regional interaction between myocardial sympathetic denervation, contractile dysfunction, and fibrosis in heart failure with preserved ejection fraction: (11)C-hydroxyephedrine PET study. Eur J Nucl Med Mol Imaging. 2017;44(11):1897-905. doi:10.1007/s00259-017-3760-y.

17. Szabó PL, Dostal C, Pilz PM, Hamza O, Acar E, Watzinger S, Mathew S, Kager G, Hallström S, Podesser BK. A. K. Remote Ischemic Perconditioning Ameliorates Myocardial Ischemia and 
Reperfusion-Induced Coronary Endothelial Dysfunction and Aortic Stiffness in Rats. J Cardiovasc Pharmacol Therap. 2021;26(6):702-13. doi:10.1177/10742484211031327.

18. Szabó PL, Ebner J, Koenig X, Hamza O, Watzinger S, Trojanek S, Abraham D, Todt H, Kubista H, Schicker K, Remy S, Anegon I, Kiss A, Podesser BK, Hilber K. Cardiovascular phenotype of the Dmd(mdx) rat - a suitable animal model for Duchenne muscular dystrophy. Dis Models Mech 2021;14(2). doi:10.1242/dmm.047704.

19. Gardner RT, Wang L, Lang BT, Cregg JM, Dunbar CL, Woodward WR, Silver J, Ripplinger CM, Habecker BA. Targeting protein tyrosine phosphatase sigma after myocardial infarction restores cardiac sympathetic innervation and prevents arrhythmias. Nat Commun. 2015;6:6235. doi:10.1038/ncomms7235.

20. Zhou L, Filiberti A, Humphrey MB, Fleming CD, Scherlag BJ, Po SS, Stavrakis S. Low-level transcutaneous vagus nerve stimulation attenuates cardiac remodelling in a rat model of heart failure with preserved ejection fraction. 2019;104(1):28-38. doi:10.1113/ep087351.

21. Sabbah HN. Electrical vagus nerve stimulation for the treatment of chronic heart failure. Cleve Clin J Med. 2011;78(0 1):24-9. doi:10.3949/ccjm.78.s1.04. Suppl 1 ) .

22. van Weperen VYH, Vos MA, Ajijola OA. Autonomic modulation of ventricular electrical activity: recent developments and clinical implications. 2021;31(6):659-676. doi:10.1007/s10286-021-00823-4.

23. Rijnierse MT, Allaart CP, de Haan S, Harms HJ, Huisman MC, Wu L, Beek AM, Lammertsma AA, van Rossum AC, Knaapen P. Sympathetic denervation is associated with microvascular dysfunction in non-infarcted myocardium in patients with cardiomyopathy. European heart journal Cardiovascular Imaging. 2015;16(7):788-98. doi:10.1093/ehjci/jev013.

24. Parati G, Esler M. The human sympathetic nervous system: its relevance in hypertension and heart failure. European heart journal. 2012;33(9):1058-66. doi:10.1093/eurheartj/ehs041.

25. Fukuda K, Kanazawa H, Aizawa Y, Ardell JL, Shivkumar K. Cardiac innervation and sudden cardiac death. Circulation research. 2015;116(12):2005-19. doi:10.1161/circresaha.116.304679.

26. Cain ME. Impact of denervated myocardium on improving risk stratification for sudden cardiac death. Trans Am Clin Climatol Assoc. 2014;125:141-53. discussion 153.

27. Siebert JR, Conta Steencken A, Osterhout DJ. Chondroitin sulfate proteoglycans in the nervous system: inhibitors to repair. Biomed Res Int. 2014;2014:845323. doi:10.1155/2014/845323.

28. Simões MV, Barthel P, Matsunari I, Nekolla SG, Schömig A, Schwaiger M, Schmidt G, Bengel FM. Presence of sympathetically denervated but viable myocardium and its electrophysiologic correlates after early revascularised, acute myocardial infarction. European heart journal. 2004;25(7):551-7. doi:10.1016/j.ehj.2004.02.016.

29. Fernandez SF, Ovchinnikov V, Canty JM Jr, Fallavollita JA. Hibernating myocardium results in partial sympathetic denervation and nerve sprouting. American journal of physiology Heart circulatory physiology. 2013;304(2):H318-27. doi:10.1152/ajpheart.00810.2011.

30. Parrish DC, Francis Stuart SD, Olivas A, Wang L, Nykjaer A, Ripplinger CM, Habecker BA. Transient denervation of viable myocardium after myocardial infarction does not alter arrhythmia 
susceptibility. 2018;314(3):H415-h423. doi:10.1152/ajpheart.00300.2017.

31. Gadioli LP, Miranda CH, Pintya AO, de Figueiredo AB, Schmidt A, Maciel BC, Marin-Neto JA, Simões MV. The severity of ventricular arrhythmia correlates with the extent of myocardial sympathetic denervation, but not with myocardial fibrosis extent in chronic Chagas cardiomyopathy: Chagas disease, denervation and arrhythmia. J Nucl Cardiol. 2018;25(1):75-83. doi:10.1007/s12350-0160556-6.

32. Chen G, Thakkar M, Robinson C, Doré S. Limb Remote Ischemic Conditioning: Mechanisms, Anesthetics, and the Potential for Expanding Therapeutic Options. Front Neurol. 2018;9:40. doi:10.3389/fneur.2018.00040.

33. Schmidt MR, Redington A, Bøtker HE. Remote conditioning the heart overview: translatability and mechanism. Br J Pharmacol. 2015;172(8):1947-60. doi:10.1111/bph.12933.

34. Gho BC, Schoemaker RG, van den Doel MA, Duncker DJ, Verdouw PD. Myocardial protection by brief ischemia in noncardiac tissue. Circulation. 1996;94(9):2193-200. doi:10.1161/01.cir.94.9.2193.

35. Paulus WJ, Tschöpe C. A novel paradigm for heart failure with preserved ejection fraction: comorbidities drive myocardial dysfunction and remodeling through coronary microvascular endothelial inflammation. J Am Coll Cardiol. 2013;62(4):263-71. doi:10.1016/j.jacc.2013.02.092.

36. Akiyama T, Yamazaki T. Myocardial interstitial norepinephrine and dihydroxyphenylglycol levels during ischemia and reperfusion. Cardiovascular research. 2001;49(1):78-85. doi:10.1016/s00086363(00)00219-4.

37. White IA, Gordon J, Balkan W, Hare JM. Sympathetic Reinnervation Is Required for Mammalian Cardiac Regeneration. Circ Res. 2015;117(12):990-+. doi:10.1161/Circresaha.115.307465.

\section{Figures}




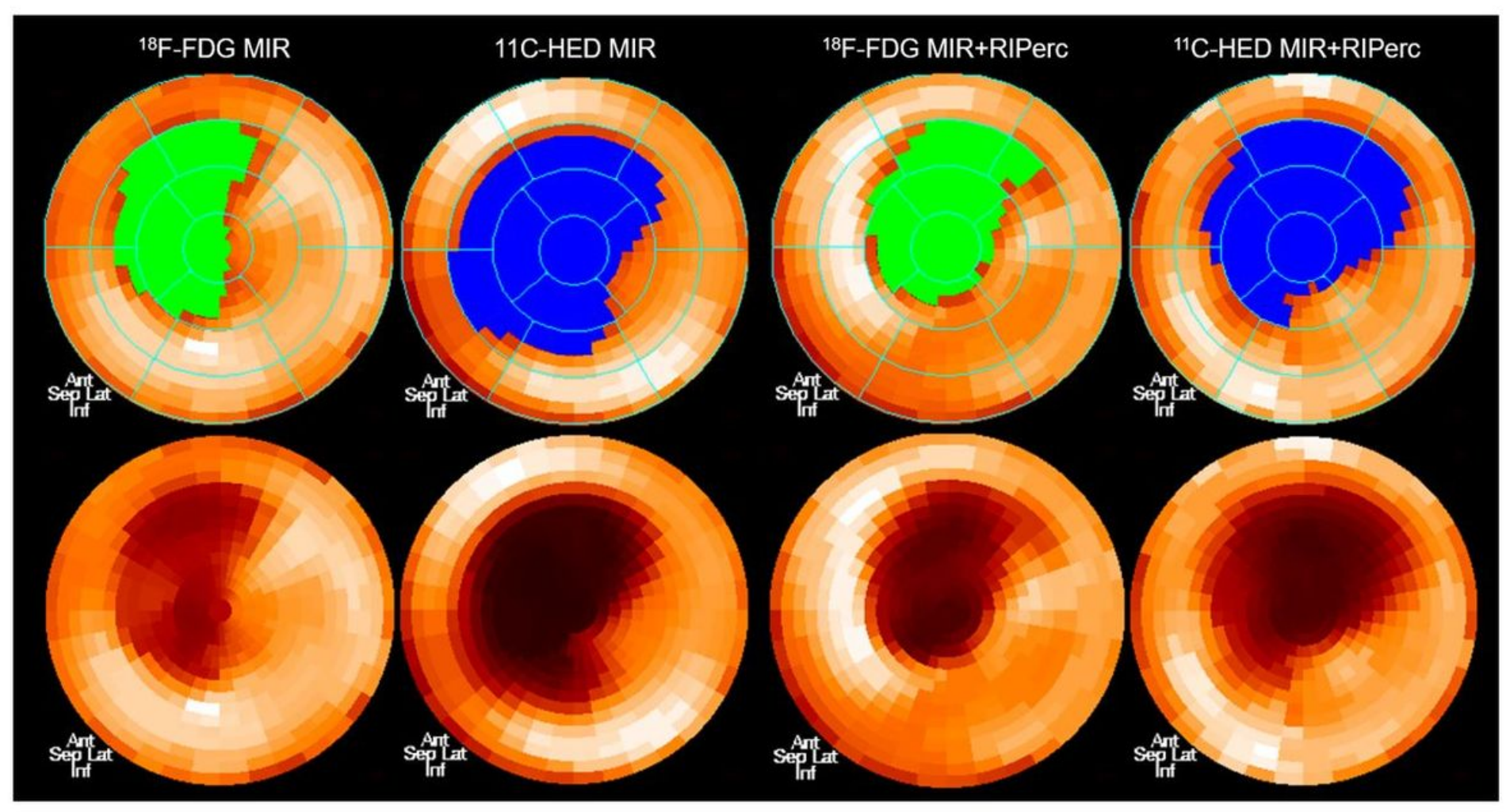

\section{Figure 1}

Polar maps of myocardial viability and sympathetic denervation were measured with 2-[18 F]FDG, and $\left[{ }^{11} \mathrm{C}\right]$ meta-Hydroxyephedrine $\left(\left[{ }^{11} \mathrm{C}\right] m \mathrm{HED}\right)$, respectively. Representative animal MIR and MIR+RIPerc present the polar maps showing larger denervation volume (downregulated $\left[{ }^{11} \mathrm{C}\right] m \mathrm{HED}$ uptake) than infarct volume (defected 2-[18 F]FDG uptake). In contrast, the rat with MIR presents polar maps showing relatively increased denervation volume $\left(\left[{ }^{11} \mathrm{C}\right] m \mathrm{HED}\right)$ and uptake ratios than the rat with RIPerc. 

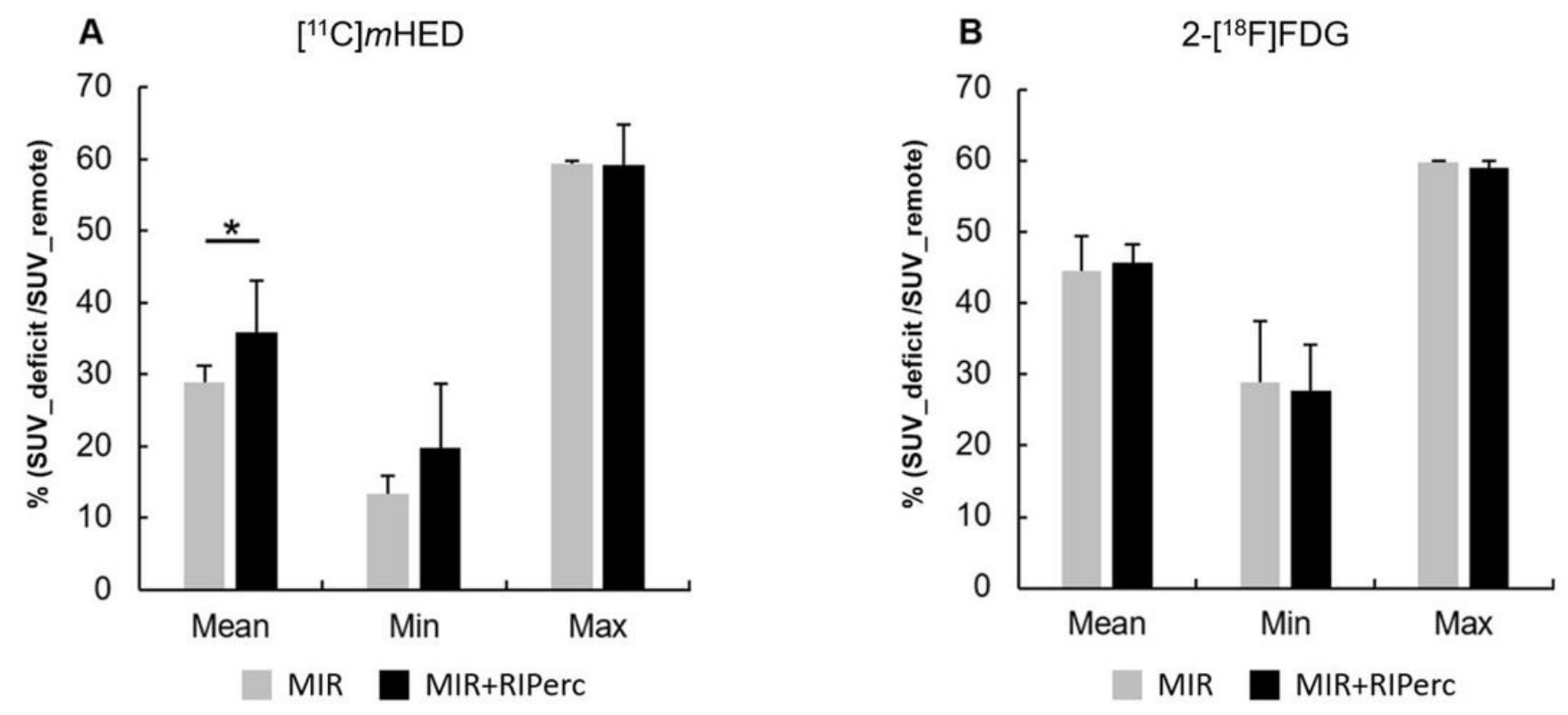

Figure 2

The average, minimum and maximum cardiac uptake ratio of the tracer deficit areas (mean, min, max) to the remote area (SUVmean) as a percentage of the left ventricular short-axis slice. The average of $\left[{ }^{11} \mathrm{C}\right] m \mathrm{HED}$ uptake defect was significantly higher in rats with RIPerc than rats with MIR $\left({ }^{*} \mathrm{p}<0.05\right)$. There is no significant difference in $2-\left[{ }^{18} \mathrm{~F}\right] \mathrm{FDG}$ uptake in the deficit areas between the two groups of rats. 


\section{MIR}
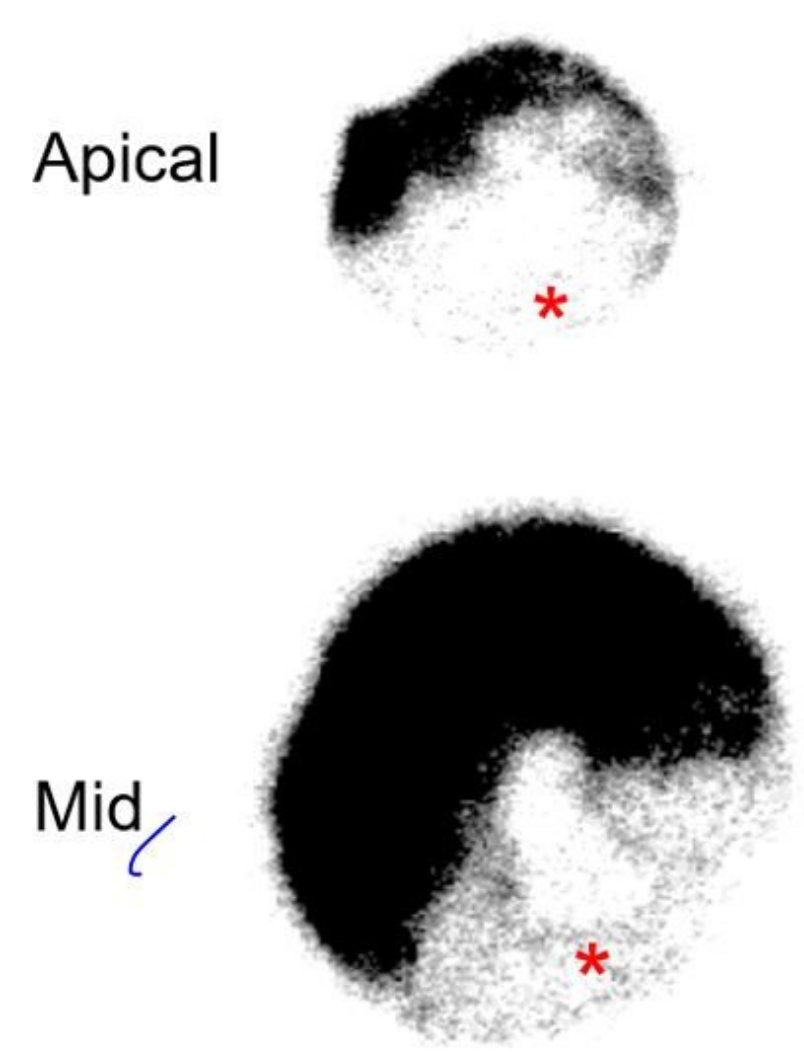

RIPerc
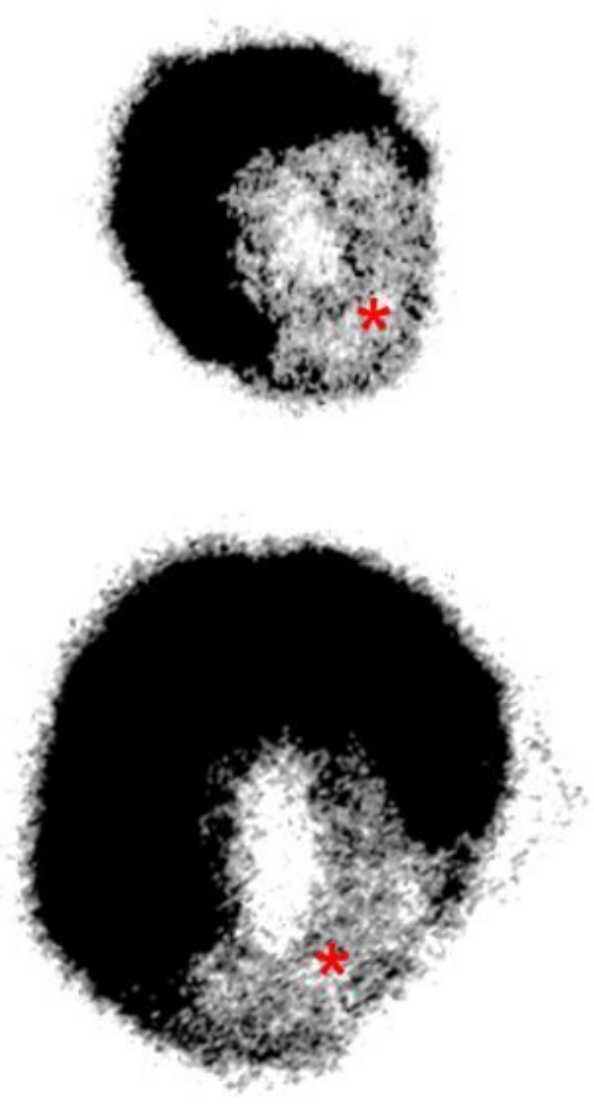

Figure 3

Autoradiography of $\left[{ }^{11} \mathrm{C}\right] m \mathrm{HED}$ in representative rats with MIR and RIPerc. Relatively increased sympathetic innervation was found in the defect area of rats with RIPerc compared to rats with MIR (*). 


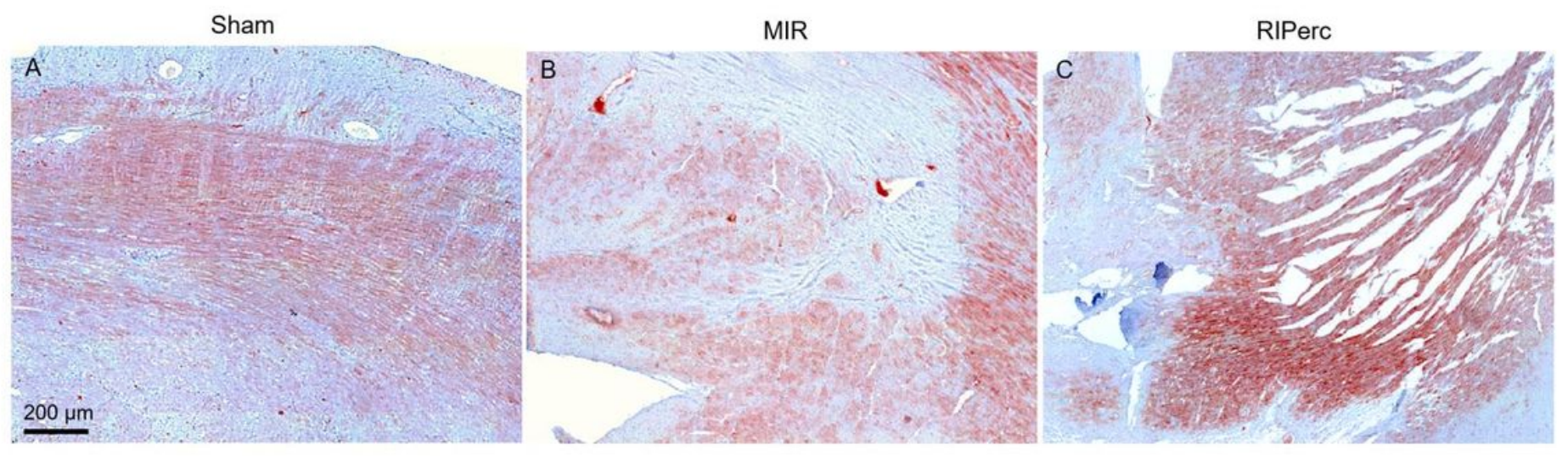

Figure 4

Immunochemistry of tyrosine hydroxylase positive neurons in the myocardium. Representative images showing tyrosine hydroxylase staining in the anterolateral area of a sham rat (A), and the defect area in the left ventricular wall in MIR (B) and RIPerc (C) rats. TH-positive cells are extensively reduced in MIR rats after myocardial infarction compared to Sham as well as less reduction in RIPerc rats.
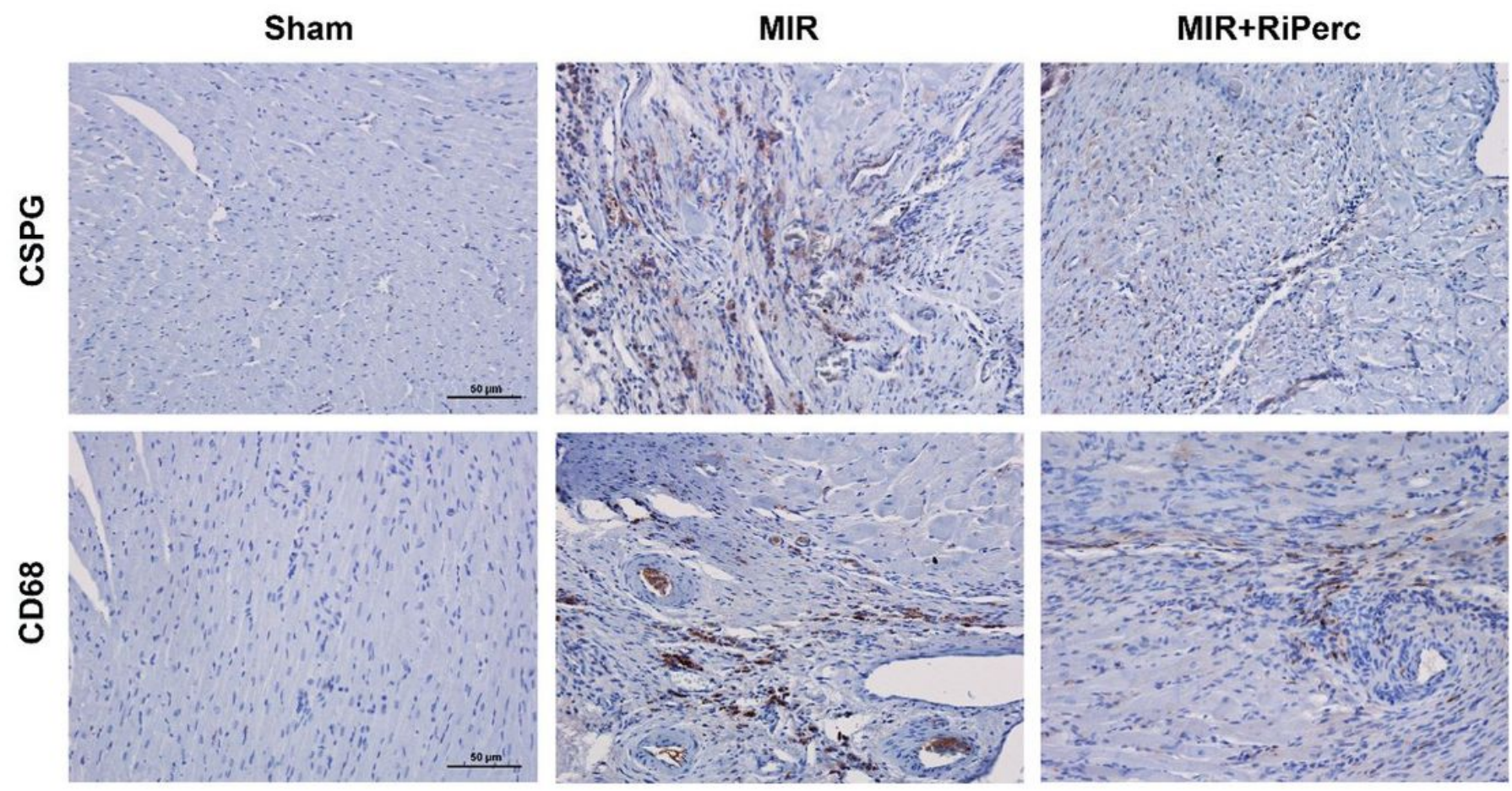

Figure 5 
Effect of remote ischemic perconditioning on influx of CD68+ cells, and CSPG expression. Representative images from each group of rats, showing (up) CSPG and (down) CD68+ macrophages in the reperfused myocardium (magnification $200 x$ and $400 x$, scalebar: $50 \mu \mathrm{m}$, respectively). 